\title{
REVISIONES
}

\section{Educación y neurociencias. La conexión que hace falta}

\author{
Education and neurosciences. The needed connection
}

\section{Educação e neurociência. A necessária conexão}

\author{
Ricardo Puebla1 , M. Paz Talma ${ }^{2}$ \\ ${ }^{1}$ Facultad de Educación, Pontificia Universidad Católica de Chile. \\ Fono: 02-3545311; e-mail: rspuebla@uc.cl \\ ${ }^{2}$ Escuela de Fonoaudiología, Facultad de ciencias de la Salud, Universidad de Talca. \\ Fono: 071-201632; e.mail: mtalma@utalca.cl
}

\begin{abstract}
RESUMEN
Para dimensionar la enseñanza, ¿es necesario saber cómo actúa nuestro cerebro cuando aprendemos? Las Neurociencias de la Educación están dando razones de peso para afirmar lo aquí preguntado. Sin embargo, Educación y neurociencias no comparten solo desde una apreciación investigativa diferente el aprendizaje humano y la enseñanza; igualmente, explican a diferentes niveles de complejidad los procesos que en estos ocurren. Los neurocientíficos requieren acercarse apropiadamente a los educadores, para que estos comprendan como se puede aplicar lo que se investiga en neurociencias a la educación; y, los educadores requieren de una formación tal que les permita entender y ser parte de los cuestionamientos capaces de ser respondidos por las neurociencias expresadas desde las disciplinas educativas propiamente tales. Por esto, se postula que estas disciplinas, así como las modernas ciencias cognitivas requieren de articuladores especialmente preparados para y desde las pedagogías, promoviendo una útil y prolongada complementariedad.
\end{abstract}

Palabras clave: neurociencias de la educación, ciencias cognitivas: aprendizaje, enseñanza.

\begin{abstract}
To gauge the teaching, is it necessary to know how our brain acts when we learn? Educational Neuroscience gives enough reason to support the previous question. However, Education and Neuroscience not only have different investigative conceptions of the human learning and teaching processes; but also explain based on different levels of complexity the way how these processes occur. Neuroscientists need to approach educators appropriately in order to understand how they can apply their research discoveries to educational issues; and educators require the appropriate training to enable themselves to understand and be part of the possible answers provided by the application of neurosciences in the educational disciplines. Bases in all these, we propose that these disciplines, such as modern cognitive sciences, require specially trained articulators -from and for education- to promote a useful and long life complementarity.
\end{abstract}

Key words: educational neurosience, cognitive sciences, learning, teaching.

\section{RESUMO}

Para avaliar o ensino, é necessário saber como nosso cérebro funciona e quando aprendemos? A Neurociência da Educação corrobora a resposta afirmativa para tal questionamento. No entanto, Educação e Neurociência não compartilham apenas pesquisas investigativas sobre o processo ensino-aprendizagem do ser humano. Elas também explicam os diferentes níveis de complexidade em que esses processos ocorrem. Os neurocientistas necessitam da colaboração dos educadores para compreender e aplicar o que pesquisam em Neurociência à Educação; os educadores precisam de formação específica que lhes permitam compreender tal processo e de sentirem-se como parte das perguntas expressas a partir das disciplinas de ensino, as quais podem ser respondidas pela Neurociência. Assim, postula-se que estas disciplinas, assim como as modernas ciências cognitivas, requerem articuladores especialmente preparados para promover uma útil e prolongada complementação das duas ciências.

Palavras chave: neurociência da educação, ciências cognitivas: aprendizagem, ensino. 


\section{INTRODUCCIÓN}

Las ciencias de la educación disciplinariamente han incorporado desde hace ya un tiempo muchos de los hallazgos que las ciencias cognitivas han establecido para tratar e investigar el fenómeno del aprendizaje y la enseñanza en las personas, encontrando repetidas veces que las apreciaciones respecto al tratamiento de la información y los procesos mentales propios de la interpretación cognitiva tienen directa aplicación en disciplinas propiamente educativas tales como el diseño curricular, la didáctica y la evaluación de los aprendizajes (Alvarez Méndez, 2001; Stufflebeam, 2001; Castillo Arredondo, 2005; Román Pérez \& Diéz López, 1988; Coll, 1995; Pozo, 1996). Asimismo, quizás si esperando repetir esta feliz coalición, los educadores y los investigadores en las disciplinas educativas mantienen una permanente expectativa $-\mathrm{y}$ gran credibilidad - respecto de la posible "gran" contribución que a la educación pueda hacer el estudio de las neurociencias (Goswami, 2006; Fischer, 2009). Pero, ¿cuál es hasta el momento la real dimensión de aporte neurocientista a la educación, desde su hacer investigativo? ¿Están claras, o realmente existen, las preguntas que la educación tiene que hacer a las neurociencias, para que esta última adquiera la trascendencia que las ciencias cognitivas tienen ya hoy en día en las ciencias de la educación?

Es pertinente señalar aquí que esta situación no ocurre de la misma forma entre las ciencias cognitivas, cuyo fin primigenio fue el plantearse explicaciones válidas y confiables acerca de la inteligencia desde el procesamiento de la información en sistemas organizados de los cuales las personas son agentes cognitivos destacados, y las neurociencias, donde las propuestas de la cognición encontraron un soporte efectivo para validar y ampliar muchos de sus hallazgos. Con la aparición del conexionismo (Glaría, 2000), las neurociencias se acercaron a la cognición, y han contribuido de manera fundamental a ampliar la significatividad del estudio del funcionamiento cerebral en seres humanos, en relación a la forma de cómo se llevan a cabo los diversos tipos de procesamiento de información a partir de modelos cognitivistas que se plantean utilizando como base, por ejemplo, la teoría de los Sistemas Dinámicos (van Gelder, 1998), o el procesamiento en paralelo de la información en la memoria de trabajo (Baddeley, 2003; Prabhakaran, Narayanan, Zhao, \& Gabrieli, 2000).

Es decir, tanto para las ciencias cognitivas como para las neurociencias desde sus propios modelos, es aceptado un mutuo sinergismo en muchas instancias de cooperación, dado que están buscando explicaciones para las cuales el objeto a ser conocido es de mutua aceptación e identificación; esto es, las representaciones mentales como procesos emergentes y los resultados del funcionamiento cerebral que permite que estos ocurran (Churchland \& Sejnowski, 1988; Rakic, 2004; McClelland \& Patterson, 2002a; McClelland \& Bybee, 2007).

\section{ANTECEDENTES DESDE LAS CIENCIAS COGNITIVAS Y LAS NEUROCIENCIAS ACERCA DE SU ENCUENTRO}

Permítasenos plantear antes que nada una observación que nos parece importante: Las ciencias cognitivas no requieren necesariamente de los arreglos neuronales -redes, tejidos, circuitos, núcleos o sistemas neuronales- para elaborar explicaciones acerca de la forma 
como se puede procesar la información en un agente de tipo cognitivo. Sin embargo, los estudios del comportamiento animal y humano han establecido que unos u otros, pueden ser vistos como agentes cognitivos por esencia, ya que, utilizan disposiciones neuronales para operar los mecanismos en los que se realiza el procesamiento de la información. Es decir, son modelos cognitivos naturales sin manipulación o programación intencionada para realizar estos procesos.

Pero el intercambio de miradas entre las dos ciencias no comenzó así tan explícitamente. Desde la década de los 40 del siglo pasado -y durante unos treinta años-, los estudiosos del comportamiento intentaron encontrar respuestas al dilema de la inteligencia, amparados por la hipótesis computacional de la mente o cognitivismo simbólico (van Gelder, 1998). Ésta se sustentaba en la idea de los arreglos simbólicos que, lógica y jerárquicamente secuenciados, y en base a las características o propiedades de los símbolos mismos, podían resolver eventos de tratamiento de datos cuyos resultados demostraban operatorias sencillas y rápidas semejantes a los procesos de análisis básicos que era capaz de realizar un individuo (Newell \& y Simon, 1976; Newell, 1980). Sin embargo, a fines de los años 60 y comienzos de los 70, se hizo evidente que las explicaciones basadas en la idea de un procesamiento cognitivo de orden digital únicamente dejaba mucho margen para la variedad de respuestas y adecuaciones -inmediatas y mediatasque hacían más difícil el estudio de los sistemas cognitivos de los seres vivos, animales y seres humanos. Las mismas neurociencias criticaban, y con razón, desde sus propios hallazgos, las características que definían este tipo de procesamiento de la información (Branquinho, 2001; Bähr, 2006; Ormrod, 2005; Varela, 1990).

El desarrollo de la idea del conexionismo actuó como puente en este caso. Surgiendo unos diez años después de que el cognitivismo representacional o simbólico hiciera su espectacular aparición, el conexionismo postuló cómo la información podía llegar a transformarse en una operatoria de un sistema de redes (por ejemplo, redes neurales (Schöner \& Kelso, 1988) en las cuales no hay una jerarquización del procesamiento de la información en sí. Esto, porque lo que no se necesita es información, sino un tratamiento de señales propias de la red que dependen de la actividad acotada de los puntos que la conforman. Es decir, unidades de red o componentes que realizan operaciones recursivas de "señales de datos" que pueden ser operadas con procesos lógicos, para lo que se necesita únicamente -dadas las propiedades de los mismos componentes de la red- que los nodos de la misma contengan operaciones restrictivas ante el paso de las señales de información que circula por ésta, filtrando las posibilidades de operación de la red ante las señales de entrada que se reciben.

Así, sin la necesidad de incorporar a las redes porciones de información, lo que éstas procesan son sus propias señales, de lo que se espera que surjan respuestas lógicas derivadas de un código operacional que contiene la misma red como atributo. Entre las características más notables de estas redes está la propiedad de que en ellas pueden ocurrir procesamientos emergentes como resultado de su propia operatoria, presentando una capacidad auto-organizativa que no podía darse en los diseños computacionales que se presentaban en los ejemplos de la cognición simbólica. Los sistemas de redes conectivas surgieron propiamente de la observación de los sistemas neurales, y su analogía supone que al presentar a la red una sucesión de patrones con puntos de entrada (unidades sensoriales), el procesamiento se reforzará cada vez que se activen las pre y post conexiones al unísono (modelo de circuito de Hebb), generando con esto un principio de operación 
recursiva o "aprendizaje". Las respuestas, fruto de este procesamiento, pueden ser dirigidas estableciendo las restricciones ya mencionadas a los nodos de la red. Después del período de aprendizaje, al presentar nuevamente el mismo patrón al sistema de red, éste lo reconocerá y repetirá la operatoria que ya ha podido "ensayar" previamente. La investigación en torno a los ejecutores centrales en el cerebro es un buen ejemplo de este paso que permitió establecer puentes de relación muy estables entre las neurociencias y la psicología cognitiva.

Los ejecutores centrales son considerados como mecanismos separados de procesos cognitivos que cumplen un papel fundamental en éstos y se encuentran en diversas partes del sistema nervioso, preferentemente en la corteza prefrontal. Al ser dañadas estas regiones de corteza se reduce notablemente la capacidad de adaptación del individuo a la influencia del medio ambiente y se tiende a la demostración de conductas muy estereotipadas. Los neurocientistas han encontrado que estos ejecutores centrales están repartidos en varias partes del cerebro, aunque la importancia de la corteza prefrontal está demostrada (Fuster, 1997 (3a. edición)).

De igual manera, la consideración del estudio de la memoria es otro buen ejemplo de una propuesta que pasó de ser una hipótesis centrada en la disposición algorítmica de secuencias de datos a ser internalizados, para transformarse en una acción emergente de ocurrencia circuital en núcleos y centros de procesos de distribución serial y jerarquizada, los que se han determinado utilizando variadas técnicas de registro entre las cuales han destacado las de neuroimágenes funcionales (Ballesteros, 1999; Hardt, Einarsson, \& Nader, 2010; Gabrieli, 1998).

Es decir, el conexionismo centró la discusión cognitiva en el entendimiento de las relaciones que establecen entre sí los símbolos más que en las características de los símbolos por sí mismos, permitiendo establecer explicaciones causales de un orden superior para el cognitivismo, donde las explicaciones emergentes (es decir, las que intentan dar cuenta de resultados no explicados por las características de los componentes que conforman las partes de un todo) pueden ser aceptadas; y, fue esta base de explicación, en conjunto con la visión de los Sistemas Complejos, lo que dio origen al estudio de los Sistemas Dinámicos (van Gelder, 1998; Martin, 2007), donde la psicología cognitiva tuvo sus puntos de encuentro más determinantes con las neurociencias ${ }^{1}$. Esta propuesta es aún muy investigada, y es posible reconocer en ella atributos que sabemos son compartidos por las redes neurales (Hinton, 1992; Haykin, 2007; Willingham, Salidis, \& Gabrieli, 2002).

En este punto, puede ser igualmente necesario puntualizar que las neurociencias cognitivas tienen varias áreas de especialidad ya declaradas, entre las cuales podríamos señalar algunas que han hecho una gran contribución a las ciencias cognitivas: la

\footnotetext{
Los Sistemas Dinámicos, sin embargo, no son fruto de la investigación cognitiva o neurocientista. Ellos surgieron de la necesidad de establecer explicaciones en el mundo natural para fenómenos complejos, que emergen del procesamiento conjunto de unidades múltiples que entran en relación ante una o más circunstancias dadas, y que no pueden ser explicados basándose solamente en el conocimiento que pueda tenerse de los componentes que conforman ese sistema (Johnson, 2001). Es decir, fenómenos típicamente relacionados con la máxima "el todo es más que la suma de sus partes", recurso que se ha mantenido como ambigua explicación para fenómenos de este tipo. Son comunes los eventos emergentes de este tipo estudiados en climatología, geología, evolución biológica, al igual que en neurociencias y en psicología social.
} 
neuropsicología, disciplina a la que hay que hacer mención en la investigación de los ejecutores centrales y cuyo objeto de estudio aporta en buenas cuentas a la arquitectura funcional de los procesos cognitivos (Coltheart, 2010); y los análisis en neuroimágenes funcionales, cuyo objeto de estudio es precisamente intentar contrastar -utilizando los modelos cognitivos- las bases neurales que posibilitan su ocurrencia en el cerebro y aportar a especificar aún más el propio diseño de los modelos testeados, como ocurrió con la investigación de la memoria más arriba esbozada (Caplan, 2009).

\section{LAS CIENCIAS DE LA EDUCACIÓN Y LAS NEUROCIENCIAS, EL FUTURO QUE VIENE. ¿CÓMO LO ADECUAMOS?}

Desde lo que es tradicional, las neurociencias -especialmente las ligadas a la cognición y al estudio de las emociones- aún continúan experimentando en modelos de tipo cognitivo, contestando preguntas del tipo: ¿Cómo la memoria, la percepción, el razonamiento y la emoción están representados en el cerebro? ¿Cuál es la interrelación que ocurre entre las emociones y la cognición? ¿Cómo se regula la conducta social en el cerebro? ¿Hasta qué punto la cultura influye en la biología del individuo o modifica las redes y núcleos neuronales? ¿La cognición humana es un proceso modular o global? ¿Cómo los cambios en el desarrollo de las personas afectan los procesos cognitivos y emocionales? Pero, descontando el apoyo que han brindado a la educación especial, a la educación la han impactado más desde lo informal como disciplina aplicada, estableciendo continuamente lazos débiles entre los productos de investigaciones no necesariamente establecidos para esta última y el hacer en las salas de clases, con resultados muchas veces pobres o sin sentido (Goswami, 2006; Fischer, 2009; Puebla Wuth, y otros, 2009). Con frecuencia, los intentos de acercamiento desde las disciplinas neurocognitivas han sido irregulares, más bien esporádicos (Meltzoff, Kuhl, Movellan, \& Sejnowsky, 2009), intentando demostrar cautamente que es posible establecer puentes de interacción entre la educación y las neurociencias (Bruer, 1997; Bruer, 1998a; Bruer, 1998b; Cameron \& Chudler, 2003; Dicket, 2005; Puebla Wuth, 2009), destacando sobre todo la ayuda y el apoyo a la educación a "servir" como agente investigado más que como participante activo en la investigación.

En los últimos quince años, se ha generado un movimiento intenso causando un gran interés desde la academia, hasta el punto en que la Organización Económica Europea (OECD) ha promovido su pertinencia y fomentado en los países desarrollados la inclinación de la balanza de la investigación neurocientífica cognitiva a favor de atender las solicitudes de la educación para el desarrollo de la enseñanza y el aprendizaje en el mundo actual (OECD, 2007). Centros de estudios de connotada reputación, como la Escuela de Educación de la Universidad de Harvard y su programa Mind, Brain and Education (http://www.gse.harvard.edu/academics/masters/mbe/), el Centro para las Neurociencias en Educación de la Universidad Cambridge (http://www.educ.cam.ac.uk/centres/neuroscience/) y el instituto Max Planck en Alemania (http://imprs-neurocom.mpg.de/imprs/index.html) están realizando procesos de enseñanza e investigación en neurociencias muy serios, con foco en la educación tradicional como disciplina propiamente tal.

Ante la emergencia de este foco investigativo, cabe preguntarse ¿cuáles son las preguntas que a la educación como ciencia y cómo disciplina en realidad interesan desde 
las neurociencias? Para contestar esta pregunta, debemos considerar primeramente que el objeto a ser investigado por la educación no necesariamente es el mismo que el que puede ser analizado desde las neurociencias; y, por tanto, el método de investigación pudiera ser también diferente. Para los investigadores educacionales, las Neurociencias aplican un modelo investigativo propio de las "ciencias médicas", cuya aproximación al fenómeno es de tipo positivista y cuantitativo, mientras que en educación muchas veces lo que se requiere son modelos de investigación centrados en perspectivas cualitativas de observación y análisis interpretativo o de metodologías cuantitativas referidas a tratamientos estadístico descriptivos y, a lo más, correlaciónales (Ruthven, Laborde, Leach, \& Tiberghien, 2009; Goswami, 2008) donde lo que resalta es la apropiada descripción, el análisis a fondo y la posterior interpretación de un fenómeno, para enmarcarlo dentro de explicaciones que no alejen en demasía al objeto que está siendo investigado de su vinculación con el medio o las circunstancias relacionales que lo han establecido. Dentro de las áreas de investigación neurocientíficas, las preguntas que se discuten y los problemas en que se trabaja abarcan principalmente las expresadas en el primer párrafo de esta sección, y su metodología tiene más significancia desde la necesidad de retirar el objeto investigado de su medio natural, para establecer con él un modelo de análisis que incluya las variables que principalmente han determinado su ocurrencia. ¿Cómo hacer, entonces, para que las neurociencias se apliquen a las preguntas que tienen interés para la educación?

Debemos dejar de lado las estimaciones ambiguas sobre la influencia de mitos pseudocientíficos que se han "enclavado" en el hacer de la profesión educativa desde una mirada ligera de las neurociencias, los que han ocurrido justamente por no tener preguntas determinantes u objetos de investigación claramente definidos desde la educación para ser respondidas desde las disciplinas neurocientíficas (Goswami, 2006; Ansari \& Coch, 2006; OECD, 2007).

Aunque se hayan dado ya grandes pasos desde las ciencias cognitivas para aportar realmente al ejercicio de la profesión educativa, y confirmado o apoyado con certeras miradas investigativas estos adelantos de la cognición con las neurociencias, nada asegura que las serias contribuciones que se han hecho hasta ahora desde estas disciplinas vayan a tener aplicación o repercusión directa sobre las disciplinas propias de la educación. Se han hecho descubrimientos impresionantes en neurociencias cognitivas respecto a la escritura (Hills, Wityk, Barker, \& Caramazza, 2002), la lectura (McCandliss \& Noble, 2003) y las matemáticas (Dehaene \& Cohen, 1995; Dehaene, Molko, Cohen, \& Wilson, 2004). Se han reconocido principios muy importantes respecto a la influencia de la ansiedad por aprender (emociones y cognición) (Fernández-Pinto, López-Pérez, \& Márquez, 2008; Gallese V., 2004; Carr, Iacoboni, Dubeau, Mazziotta, \& Lenzi, 2003) y los déficit atencionales (Sonuga-Barke \& Sergeant, 2005; Hale, Bookheimer, McGough, Pillips, $\&$ McCracken, 2007), probablemente uno de los principales factores que influyen en la calidad de lo que se aprende; se han establecido procesos de interacción comunicativa donde juegan roles trascendentales de entendimiento y comprensión de la acción los gestos manuales (Kelly, Manning, \& Rodak, 2008; Kelly, Ward, Creigh, \& Bartolotti, 2007), la corporalidad (Curby \& Gauthier, 2010) y las expresiones faciales (Blair, 2003; Adolphs, Damasio, Tranel, \& Damasio, 1996), asociando estas demostraciones a áreas de la corteza cerebral que procesan información desde la percepción hasta su asociación con la experiencia de las personas. 
Sin embargo, para las ciencias de la educación postulamos que estos hallazgos irán encontrando sentido a futuro, solo cuando se acerquen a las disciplinas neurocientíficas investigadores educacionales que puedan contestar preguntas tales como: ¿Cuán contemporáneas o pertinentes son las teorías educacionales y los métodos didácticos ante los más recientes hallazgos de las neurociencias cognitivas? ¿Es necesario o útil para el desarrollo de la teoría educativa o la generación de políticas educacionales entender la estructura y función del cerebro? ¿Pueden las neurociencias identificar las diferencias en el desarrollo que deben ser atendidas por las diferentes técnicas didácticas? ¿Son observables, a través de la estructura o funciones cerebrales, las diferencias individuales que existen en el aprendizaje? ¿Es posible que el diseño de la evaluación de los aprendizajes se vea beneficiado con el conocimiento de la función cerebral? ¿Es un aporte al currículo educativo, el conocer las características del desarrollo cerebral de las personas? La capacidad de las neurociencias aplicadas a la educación de contestar a estas y otras preguntas que son demandas permanentes de la educación como disciplina, determinará a futuro su valor en el ejercicio de lo educativo. Pero los neurocientíficos como tales están aún lejos de poder acercarse sin más a intentar resolver los problemas educativos dado que, como se ha señalado antes, aún su principal foco de estudio son las representaciones mentales. Desde las neurociencias, deben acercarse a la educación especialistas en neurociencias con experiencia docente, que puedan interactuar con educadores transmitiendo los hallazgos reales de la disciplina neurocientífica, ayudando a retirar los neuromitos de las creencias de los educadores (Goswami, 2006; Fischer, 2009). De igual forma, es necesario que las inquietudes de los educadores se traduzcan en preguntas posibles de ser investigadas desde las disciplinas cognitivas y neurocognitivas, requiriendo -como menciona, ciertamente para esto Usha Goswami (2006) - la concurrencia de especialistas e investigadores en neurociencias con capacidad para poder interpretar los requerimientos de los maestros.

Pero, para poder llegar a contestar con pertinencia educativa las preguntas antes señaladas, la investigación neurocientífica debe acercarse metodológicamente a las salas de clases, y constituir modelos de investigación que puedan llevarse a cabo en terreno o en simulaciones más cercanas a la práctica educativa. Es necesario que concurran entonces hacia las neurociencias investigadores educacionales que entiendan y convivan con naturalidad tanto con las ciencias de la educación y su práctica disciplinaria en el aula, como con el ámbito neurocientista y sus modelos de investigación disciplinaria (Bruer, 1997; OECD, 2007; Fischer, 2009; Meltzer, 2007; Meltzoff, Kuhl, Movellan, \& Sejnowsky, 2009). Es decir, la disciplina de las neurociencias de la educación tiene que llegar a establecerse como tal, incorporándola oficialmente en los currículos de formación de educadores y configurándola en el concierto de las especialidades del ámbito educativo ya sea como diplomados de especialidad, maestrías o focos de acción investigativa en las propuestas doctorales en educación.

\section{REFERENCIAS BIBLIOGRÁFICAS}

Adolphs, R., Damasio, H., Tranel, D. \& Damasio, A.R. (1996). Cortical Systems for Recognition of Emotion in Facial Expression. The Journal of Neurosciences, 16(23), 7678-7687.

Alvarez Méndez, J.M. (2001). Entender la Didáctica, entender el Currículum. Madrid: Miño y Dávila.

Baddeley, A. (October de 2003). Working Memory: Looking Back and Looking Forward. Nature Reviews of Neuroscience, 4, 829-839. 
Bähr, M. (2006). Brain Repair. USA: Springer.

Ballesteros, S. (1999). Memoria Humana: Investigación y Teoría. Psicothema, 11(4), 705-723.

Blair, R.J. (2003). Facial expressions, their communicatory functions and neuro-cognitive substrates. Phil. Trans. R. Soc. Lond. B(358), 561-572.

Blakemore, S.J. \& Frith, U. (2005). The Learning Brain: Lessons for Education. Oxford, UK: Backwell.

Branquinho, J. (2001). The Foundations of Cognitive Science. Lisboa: Oxford University Press.

Bruer, J.T. (1997). Education and the brain: A bridge too far. Educational Researcher, 8, 4-16.

Bruer, J.T. (1998a). Brain science, brain fiction. Educational Leadership, 56, 14-18.

Bruer, J.T. (1998b). Let's put brain science on the back burner. NASSP Bulletin, 82, 9-19.

Cameron, W. \& Chudler, E. (2003). A role for neuroscientist in engaging young minds. Nature Reviews of Neuroscience, 4, 1-6.

Caplan, D. (2009). Experimental Design and Interpretation of Functional neuroimaging Studies of Cognitive Processes. Human Brain Mapping(30), 59-77.

Carr, L., Iacoboni, M., Dubeau, M.C., Mazziotta, J.C. \& Lenzi, G.L. (2003). Neural mechanisms of empathy in humans: A relay from neural systems for imitation to limbic areas. Proceedings of the National Academy of Sciences, 100(9), 5497-5502.

Castillo Arredondo, S. (2005). Compromisos de la Evaluación Educativa. Madrid: Pearson Educación.

Churchland, P.S. \& Sejnowski, T.J. (4 November 1988). Perspectives on Cognitive Neuroscience. Science, 242, 741-745.

Coll, C. (1995). Psicología y Currículum. Barcelona: Paidós.

Coltheart, M. (1 January 2010). Lessons From Cognitive Neuropsychology for Cognitive Science: A Reply to Patterson and Plaut (2009). (C.S. Society, Ed.) Topics in Cognitive Science, 2(1), 3-11.

Curby, K.M. \& Gauthier, I. (April 2010). To the Trained Eye: Perceptual Expertice Alters Visual Processing. Topics in Cognitive Science, 2(2), 189-201.

Dehaene, S., \& Cohen, L. (1995). Towards Anatomical and Functional Model of Number Processing. Mathematical Cognition, 1, 85-120.

Dehaene, S., Molko, N., Cohen, L. \& Wilson, A.J. (April 2004). Arithmetic and the brain. Current Opinion in Neurobiology, 14(2), 2018-2024.

Dicket, R. (2005). Applying the Neurosciencies to educational research: can cognitive neuroscience bridge the gap? Anual Meeting of the American Educational Research Association, (part II). Montreal, Cánada.

Dujardin, K., Derambure, P., Defebvre, L., Bourriez, J., Jacquesson, J. \& Guieu, J. (1993). Eventrelated desynchronization/ERD) during a recognition task: effect of attention. Electrorncephalogr. Clin. Neurophysiol., 86, 353-356.

Fernández-Pinto, I., López-Pérez, B. \& Márquez, M. (2008). Empatía: Medidas, teorías y aplicaciones en revisión. (S.d. Murcia, Ed.) Anales de Psicología, 24(2), 284-298.

Fischer, K.W. (2009). Mind. Brain, and Education: Building a Scientific Groundwork for Learning and Teaching. Mind, Brain and Education, 3(1), 3-17.

Fuster, J.M. (1997 (3a. edición)). The Prefrontal Cortex-Anatomy Physiology, and of Neuropsychology of the Frontal Lobe. Philadelphia: Lippincott-Raven.

Gabrieli, J.D. (February 1998). Cognitive Neuroscience of Human Memory. Annual Review of Psychology, 49, 87-115.

Gallese, V. (2004). Intentional attunement: The Mirror Neuron system and its role in interpersonal relations. Recuperado el 26 de junio de 2009, de http://www.interdisciplines.org/mirror/papers/1

Gallese, V. \& Goldman, A. (December 1998). Mirror neurons and the simulation theory of mindreading. Trends in Cognitive Sciences, 2(12), 493-501.

Glaría, A. (2000). Conexionismo: Articulador de las Neurociencias, las Psicologías de la Cognición y la Inteligencia Artificial. Revista de Psicología Universidad de Valparaíso (RevpsiUV), $1(1), 35-49$. 
Goswami, U. (2006). Neuroscience and education: from research to practice? Nature Reviews Neuroscience(7), 2-7.

Goswami, U. (2008). Principles of Learnig, Implications for Teaching: A Cognitive Neuroscience Perspective. Journal of Philosophy of Education, 42(3-4), 381-399.

Hale, S.T., Bookheimer, S., McGough, J.J., Pillips, J.M. \& McCracken, J.T. (2007). Atypical Brain Activation During Simple \& Complex Levels of Processing in Adult ADHD An fMRI Study. Journal of Attention Disorders, 11(2), 125-139.

Hardt, O., Einarsson, E.Ö. \& Nader, K. (January de 2010). A Bridge Over Troubled Water: Reconsolidation as a Link Between Cognitive and Neuroscientific Memory Research Traditions. Annual Review of Psychology, 61, 141-167.

Haykin, S. (2007). Neural Networks: A Comprehensive Foundations. Upper Saddle-River, NJ.: Prentice-Hall, Inc.

Hills, A.E., Wityk, R.J., Barker, P.B. \& Caramazza, A. (2002). Neural Regions essential for writing verbs. Nature Neuroscience, 6, 19-20.

Hinton, G.E. (1992). How neural networks learn from the experiences. Scientific American, 244-251.

Johnson, S.B. (2001). Emergence. New York: Scribner.

Kelly, S.D., Manning, S.M. \& Rodak, S. (2008). Gesture Gives a Hand to Language and Learning: perspectives from Cognitive Neuroscience, Developmental Psychology and Education. Language and Linguistic Compass, 2(4), 569-588.

Kelly, S.D., Ward, S., Creigh, P. \& Bartolotti, J. (2007). An intentional stances modulates the integration of gesture and speech during comprehension. Brain and Languages, 101, 222-233.

Martin, A. (2007). The representations of object concepts in the brain. Annual Review of Psychology(58), 25-45.

McCandliss, B.D. \& Noble, K.G. (28 August 2003). The development of reading impairment: a cognitive neuroscience model. Mental Retardation and Developmental Disabilities Research Reviews, 9(3), 196-205.

McClelland, J.L. \& Bybee, J. (2007). Gradience of Gradience: A reply to Jackendoff. The Linguistic Review, 24, 437-455.

McClelland, J.L. \& Patterson, K. (2002a). Rules or Connections in Past-Tense inflections: What does the evidence rule out? Trends in Cognitive Sciences, 11, 465-472.

Meltzer, L. (2007). Executive Function in Education. From Theory to Practice. New York: The Guilford Press.

Meltzoff, A.N., Kuhl, P.K., Movellan, J. \& Sejnowsky, T.J. (21 July 2009). Foundations for a New Science of Learning. Science, 325, 284-288.

Newell, A. (1980). Physical symbol systems. Cognitive Science, 4, 135-183.

Newell, A. \& y Simon, H. (1976). Computer science as empirical enquire. Symbols and search. Communications of the Associantion of Computing Machinery, 19, 113-126.

OECD. (2007). Understanding the Brain:The Birth of a Learning Science. París: CERI-OECD.

Ormrod, J.E. (2005). Aprendizaje humano (Cuarta ed.). Madrid, España: Pearson Educación S.A.

Pinel, J.P. (2006). Biopsicología. México, D.F.: Pearson.

Pozo, J.I. (1996). La Psicología Cognitiva y la Educación Científica. Investigações em Ensino de Ciências, 1(2), 110-131.

Prabhakaran, V., Narayanan, V., Zhao, Z. \& Gabrieli, J.D. (2000). Integration of diverse information in working memory within the frontal lobe. Nature Neuroscience, 3, 85-90.

Puebla Wuth, R.S. (Septiembre de 2009). Las Funciones Cerebrales del Aprendiendo a Aprender (Una aproximación al sustrato neurofuncional de la Metacognición). Revista iberoamericana de Educación, 50(3), 1-10.

Puebla Wuth, R.S., Elgueta Cancino, E., Caballero Moyano, P., Caparrós Manosalva, C., Gajardo Contreras, C., Urzúa Alul, A. y otros. (2009). Modelo Exploratorio para la Evaluación de Competencias Intermedias del Primer Ciclo de Pregrado en Kinesiología en la Universidad de Talca. Revista Electrónica de Desarrollo de Competencias (REDEC), 1(3), 126-160. 
Rakic, P. (2004). Introduction. Evolution and Development. En Gazzaniga, M.S., The Cognitive Neurosciences III (págs. 3-22). Cambridge, MA: MIT Press.

Román Pérez, M. \& Diéz López, E. (1988). Aprendizaje y Currículum. Diseños Curriculares Aplicados. Santiago de Chile: Federación de Instituciones de Educación Particular FIDE.

Ruthven, K., Laborde, C., Leach, J. \& Tiberghien, A. (2009). Design Tools in Didactical Research: Instrumenting the Epistemological and Cognitive Aspects of the Design ofTeaching Sequences. Educational Researcher, 38(5), 329-342.

Schöner, G. \& Kelso, J. (1988). Dynamic pattern generation in behavioral and neural systems. Science, 239, 1513-1520.

Smith, A. (1996). Accelerated learning in the Classroom. Bodmin, UK: Network Educational Press, Ltd.

Sonuga-Barke, E.J. \& Sergeant, J. (2005). The neuroscience of ADHD: multidisciplinary perspective on a complex developmental disorder. Developmental Science, 8(2), 13-14.

Stufflebeam, D. (2001). Evaluation Models. En D. Stufflebeam, New Directions for Program Evaluation. San Francisco, CA: Jossey-Bass.

Van Gelder, T. (1998). The dynamical hypothesis in cognitive science. Behavioral and Brain Sciences, $21,615-665$.

Varela, F.G. (1990). Conocer. Barcelona: Gedisa.

Willingham, D.B., Salidis, J. \& Gabrieli, J.D. (2002). Direct Comparison of Neural Systems Mediating Conscious and Unconscious Skill Learning. Journal of Neurophysiology, 88, 1451-1460.

Zull, J.F. (2002). The Art of the Changing Brain. Enrichment the Practice of Teaching by Exploring the Biologý of Learning. Virginia: Stylus Publishing, LLC. 
Las colaboraciones, suscripciones y correspondencia deben ser dirigidas a la Dirección de la revista: Oficina de Publicaciones, Facultad de Filosofía y Humanidades, Universidad Austral de Chile, Casilla 567, Fono/Fax: 5663 221275, Valdivia, Chile. E-mail: eped@uach.cl

Las suscripciones incluyen los gastos de envío:

Chile: cheque o vale vista por \$10.000. Otros países: US\$ 15 .

El canje debe ser enviado a Biblioteca Central, Universidad Austral de Chile,

Correo 2, Valdivia 

ANDROS IMPRESORES

www.androsimpresores.cl 
